\title{
O processo de amamentação do bebê pré-termo: perspectiva dos registros maternos no "diário do bebê"
}

\author{
The pre-term breastfeeding process: perspective \\ of maternal records in "baby diary"
}

\author{
Luana Cláudia dos Passos Aires ${ }^{1}$, Viviane Godoy Galhardo ${ }^{2}$, Leila Garcia de \\ Oliveira Pegoraro ${ }^{3}$, Lidiane Ferreira Schultz ${ }^{4}$, Edilaine Giovanni Rossetto ${ }^{5}$, \\ Adriana Valongo Zani ${ }^{6}$, Sarah Nancy Deggau Hegeto de Souza ${ }^{7}$
}

\section{Resumo}

Objetivo: Descrever os fatores envolvidos no processo de amamentação do bebê pré-termo internado em uma Unidade Neonatal registrados em um "Diário do bebê" preenchido pela mãe. Método: Pesquisa descritiva, prospectiva e longitudinal, com abordagem quantitativa, realizada no período de setembro de 2010 a junho de 2011 com 38 mães de recém-nascidos pré-termo internados na Unidade Neonatal. Resultados: Predomínio de mães com idade entre 20 a 34 anos, com companheiro, escolaridade maior de oito anos, renda familiar entre um a cinco salários mínimos, e não possuíam experiência prévia com aleitamento materno de outros filhos. A frequência do uso do diário foi maior ou igual a três vezes/semana; a média de número de ordenhas diárias foi maior ou igual a três e a duração da ordenha de 16 a 30 minutos. A média de volume ordenhado foi menor ou igual a $100 \mathrm{ml} / \mathrm{semana}$. Conclusão: O sucesso da amamentação do bebê pré-termo na Unidade Neonatal depende de vários fatores associados e continua sendo um desafio para as mães, profissionais de saúde e família, porém estratégias como incentivo para o registro no diário mostraram-se adequadas para sua manutenção e para a intervenção de enfermagem na prática assistencial.

Palavras-chave: Prematuro. Aleitamento materno. Relações mãe-filho. Unidades de terapia intensiva neonatal. Enfermagem neonatal.

\footnotetext{
${ }^{1}$ Doutoranda em Enfermagem na Universidade Federal de Santa Catarina (UFSC), Florianópolis, Santa Catarina, Brasil. Docente Adjunta do Curso de Graduação em Enfermagem da Faculdade IELUSC, Joinville, Santa Catarina, Brasil. E-mail: luana.aires08@gmail.com

${ }^{2}$ Residência em Enfermagem Perioperatória pela Universidade Estadual de Londrina (UEL), Londrina, Paraná, Brasil.

${ }^{3}$ Residente de Gerência dos Serviços de Enfermagem na Universidade Estadual de Londrina, Londrina, Paraná, Brasil.

${ }^{4}$ Doutoranda em Saúde e Meio Ambiente na Universidade da Região de Joinville (Univille), Joinville, Santa Catarina, Brasil. Docente Adjunta do Curso de Graduação em Enfermagem da Faculdade IELUSC, Joinville, Santa Catarina, Brasil.

${ }^{5}$ Doutorado em Saúde Pública pela Universidade de São Paulo (USP), Ribeirão Preto, São Paulo, Brasil. Professora Associada do Departamento de Enfermagem da Universidade Estadual de Londrina, Londrina, Paraná, Brasil.

${ }^{6}$ Doutorado em Saúde Coletiva pela Universidade Estadual Paulista Júlio de Mesquita Filho (Unesp), Botucatu, São Paulo, Brasil. Professora Adjunta do Departamento de Enfermagem da Universidade Estadual de Londrina, Londrina, Paraná, Brasil.

${ }^{7}$ Doutorado em Ciências pela Universidade de São Paulo, Ribeirão Preto, São Paulo, Brasil. Professora Associada do Departamento de Enfermagem da Universidade Estadual de Londrina, Londrina, Paraná, Brasil.
} 


\begin{abstract}
Objective: To describe the factors involved in the breastfeeding process of preterm infants hospitalized at a Neonatal Unit recorded in a "Baby diary" completed by the mother. Method: This is a descriptive, prospective and longitudinal study that uses a quantitative approach. The study was conducted between September 2010 and June 2011 with 38 mothers of preterm infants hospitalized at a Neonatal Unit. Results: There was prevalence of mothers aging between 20 and 34 years old and that have partners. These mothers had eight or more years of school education, family income betwen one and five mininum wages, and no previous experience in breastfeeding other children. The frequency of use of the diary was equal or greater than three times per week. The average daily breast pumpings was equal or greater than three and the duration of the breast pumping was between 16 and 30 minutes. The average expressed breast milk volume was equal or less than $100 \mathrm{~mL}$ per week. Conclusion: The success of breastfeeding of preterm infants at the Neonatal Unit depends on several associated factors and continues to be a challenge to mothers, healthcare professionals and families. However, strategies to promote registering on baby diraries seems to be adequate ir order to maintain breastfeeding and to guide interventions from nurses in the assistential practice.
\end{abstract}

Keywords: Premature. Breastfeeding. Mother-child relations. Neonatal intensive care units. Neonatal nursing.

\section{Introdução}

O Aleitamento Materno (AM) é a forma mais natural e segura de alimentar a criança, sendo recomendado exclusivamente até o sexto mês de vida. ${ }^{(1)}$ Esta prática é essencial para alcançar o crescimento e desenvolvimento infantil adequado, protegendo contra infecções, aumentando o coeficiente de inteligência, reduzindo o risco para o excesso de peso, obesidade, diabetes e doenças crônicas não transmissíveis na idade adulta. ${ }^{(2)}$ Promove beneficios para a mãe como proteção contra o câncer de mama, câncer de ovário e diabetes tipo $2,{ }^{(2)}$ além da associação com fatores econômicos, sociais,,$^{(3)}$ ambientais $^{(4)}$ e genético. $^{(5)}$

Um neonato a termo nasce com condições de se alimentar por via oral, com coordenação entre as funções de sucção, deglutição e respiração, primordiais para as suas funções vitais. ${ }^{(6)} \mathrm{Já}$ um bebê nascido pré-termo, em razão principalmente de sua imaturidade cerebral, apresenta limitações, como dificuldade de permanecer em estado de alerta, tônus predominantemente extensor e reflexos orais ausentes ou incompletos, que dificultam ou impedem a alimentação via oral nos primeiros momentos de vida. ${ }^{(7)}$
A preferência pelo AM, em especial, para neonatos pré-termo justifica-se pela redução do tempo de internação e infecção, atribuídas às propriedades imunológicas provenientes do leite humano, bem como sua ação na maturação gastrintestinal e no estabelecimento do vínculo mãe-filho. ${ }^{(8)}$

Para o binômio mãe-bebê, a permanência em uma Unidade Neonatal (UN) representa um desafio para a garantia do $\mathrm{AM}^{(9)}$ pois esta mãe deve realizar ordenha precocemente e em intervalos regulares, realizando o esvaziamento completo das mamas a fim de manter a produção láctea. ${ }^{(10)}$ Múltiplos fatores colaboram para o não sucesso do AM em nascidos pré-termo em UN, como separação da mãe e do bebê, dificuldades encontradas pelas mães para iniciar e manter a produção do leite, instabilidade clínica do lactente e sucção débil, vivência do nascimento do filho antes do tempo esperado, sentimentos de incapacidades relacionado ao cuidado com o filho, ansiedade e depressão, incertezas relacionadas à alta hospitalar, preocupações econômicas e volta ao trabalho, dinâmica familiar, cuidado com outros filhos, estratégias e assistência por profissionais de saúde não eficaz ou especializada..$^{(8,11-14)}$ 
Diante do exposto, é importante descrever estratégias facilitadoras de registro da produção do leite, sentimentos das mães durante a lactação e possíveis preditores que podem influenciar na lactação de mães de bebês pré-termos internados na Unidade Neonatal.

Acredita-se que a compreensão desses fatores favoreça para a manutenção da lactação e possa contribuir para a identificação precoce da queda na produção láctea e, desta forma, direcionar a equipe de saúde para oferecer à mãe apoio e ensinamento individualizado e multiprofissional, com implementação de estratégias eficientes na prática. Portanto, o objetivo deste estudo foi descrever os fatores envolvidos no processo de amamentação do bebê pré-termo internado em uma Unidade Neonatal registrados em um "Diário do Bebê" preenchido pela mãe.

\section{Método}

Estudo descritivo, prospectivo e longitudinal de abordagem quantitativa, desenvolvido na UN de um Hospital Universitário localizado na Região Sul do Brasil, sendo este um serviço público, credenciado pelo Sistema Único de Saúde (SUS) e considerado Centro Regional de Referência para recém-nascido pré-termo. Esta unidade possui sete leitos para cuidados intensivos neonatais e 10 leitos para cuidados intermediários neonatais.

Os critérios de inclusão foram mães de recém-nascidos pré-termo com peso inferior a $1500 \mathrm{~g}$ e/ou idade gestacional inferior a 34 semanas que participaram do projeto "Uma rede de apoio à família prematura". ${ }^{(14)}$

Como critérios de exclusão: não ter realizado nenhuma anotação no "Diário do Bebê" durante a internação de seu filho e mães de recémnascido com síndromes ou outra patologia que impossibilite condições adequadas de saúde para realizar ordenha e amamentação, e que tiveram óbito antes da primeira semana de vida.

Durante o período de coleta dos dados, setembro de 2010 a junho de 2011, e seguindo o fluxograma de recrutamento, participaram 38 mães. Foi considerado como critério para a análise dos registros maternos ter utilizado o diário por, no mínimo, uma semana. Sendo assim, não foi considerado que houve perdas nesta pesquisa, independente da continuidade do seguimento.

Figura 1 - Fluxograma de recrutamento, Londrina, 2011.
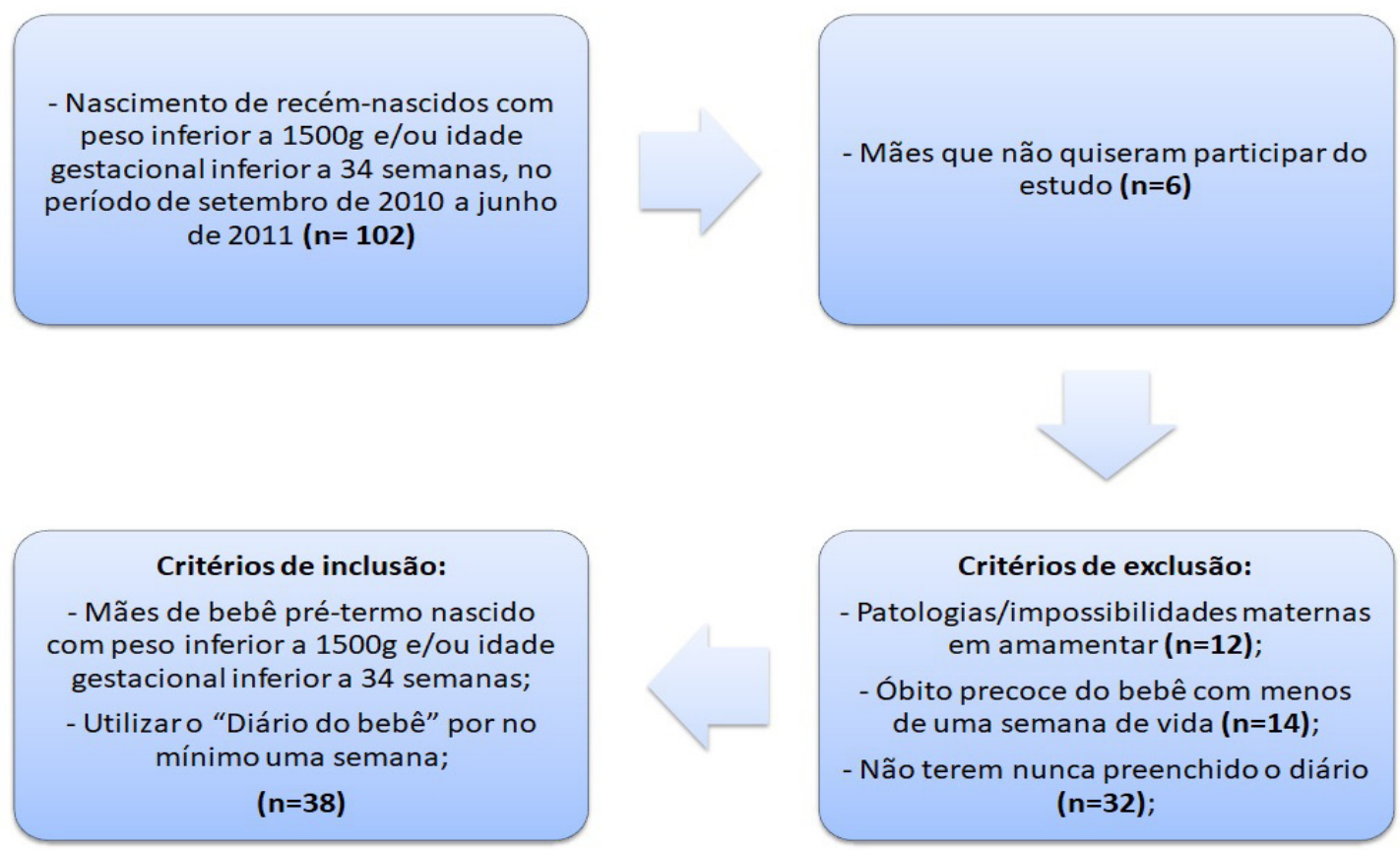

Fonte: Autoras 
O projeto de extensão "Uma rede de apoio à família prematura" tem a finalidade de prestar uma assistência individualizada e dar apoio aos familiares que vivenciam a internação de seu bebê pré-termo na instituição. ${ }^{(14)}$ Neste projeto, as enfermeiras residentes em neonatologia ficam responsáveis pelo acompanhamento semanal referente ao AM do neonato prestando assistência à mãe e à família. Aspectos importantes incluem, em cada encontro, a avaliação da produção láctea e a realização da ordenha. As mães também são orientadas e estimuladas logo após o parto e continuadamente a realizarem a ordenha, visando garantir a produção láctea.

No momento da abordagem para a participação no projeto, na primeira visita da mãe ao bebê na UN ou ainda na maternidade, entregase um manual denominado "Diário do Bebê". ${ }^{(15)}$ Esse material consiste de um instrumento elaborado pelo Programa de Residência em Enfermagem Neonatal da Universidade Estadual de Londrina, ${ }^{(14)}$ com a finalidade de ser uma tecnologia leve que favoreça o enfrentamento materno para o nascimento e internação de um filho pré-termo. Além de orientações relacionadas ao processo de internação e cuidados ao bebê pré-termo, conta com um espaço para registro de informações do bebê, fotos, carimbo de pé e mão, e o "Diário da Ordenha" que visa o registro de sua frequência, volume e tempo, além do estado emocional materno. A residente pesquisadora manteve contato direto com as mães durante todo o período de coleta de dados, orientando e incentivando o uso do diário, bem como auxiliando as mães quando evidenciadas dificuldades no registro do mesmo.

As fontes de coleta de dados da pesquisa foram: as informações referidas no "Diário do Bebê”, e os dados do prontuário da família, para caracterização dos dados sócioeconômicos e obstétrico. Como se trata de um instrumento que já é utilizado pelo Programa de Residência em Enfermagem Neonatal não foi realizado teste piloto. Os dados foram coletados desde a internação do neonato até a alta hospitalar, óbito do bebê ou durante o tempo que as mães realizaram as anotações, sendo tabulados semanalmente em uma planilha do programa Excel ${ }^{\circledR}$ para posterior análise.

As médias foram calculadas com base na soma diária da variável na semana, dividida pela frequência do uso do diário pela mãe na semana. As variáveis: frequência do uso do diário na semana; média de ordenhas realizadas ao dia; média de volume ordenhado por dia; frequência de visita da mãe ao bebê na semana; média de tempo para ordenha por dia e estado emocional materno, foram analisadas mediante a transformação em variáveis binárias e terciárias. O tempo de acompanhamento do diário do bebê foi do nascimento até sete semanas.

$\mathrm{Na}$ análise estatística descritiva foram realizadas frequências absolutas e relativas das variáveis. Referente ao sentimento predominante, correspondia à face do sentimento mais vezes assinalado na semana, sendo os mesmos classificados em sentimentos bons (feliz, tranquila) e ruins (desanimada, preocupada, ansiosa, triste).

A pesquisa foi aprovada pelo Comitê de Ética em Pesquisa, sob $\mathrm{n}^{\mathrm{o}}$ 066/2013. CAAE: 15565.2011.52 e foi conduzida de acordo com os padrões éticos nacionalmente exigidos pelas resoluções 466/2012 e 510/2016.

\section{Resultados}

Na Tabela 1 foram descritas as características das mães participantes e seus bebês $(n=38)$.

A maioria das mães participantes deste estudo tinham idade entre 20 a 34 anos, com companheiro, escolaridade maior de oito anos, renda familiar entre um a cinco salários mínimos, e não possuíam experiência prévia com aleitamento materno de outros filhos. Quanto à caracterização dos recémnascidos, destaca-se a maior frequência de idade gestacional ao nascer abaixo de 30 semanas, bem como o peso menor que $1000 \mathrm{~g}$. 
Tabela 1 - Características das mães e seus bebês nascidos pré-termos internados na Unidade Neonatal, Londrina, 2011.

\begin{tabular}{|c|c|c|}
\hline & $\mathbf{N}$ & $\%$ \\
\hline \multicolumn{3}{|l|}{ Idade da mãe } \\
\hline$\leq 19$ anos & 9 & 23,7 \\
\hline $20-34$ & 23 & 60,5 \\
\hline$\geq 35$ & 6 & 15,8 \\
\hline \multicolumn{3}{|l|}{ Estado civil } \\
\hline Com companheiro & 35 & 92,1 \\
\hline Sem companheiro & 3 & 7,9 \\
\hline \multicolumn{3}{|l|}{ Escolaridade } \\
\hline$<8$ anos & 7 & 18,4 \\
\hline$\geq 8$ anos & 31 & 81,6 \\
\hline \multicolumn{3}{|l|}{ Renda familiar } \\
\hline$<1$ salário mínimo & 11 & 28,9 \\
\hline$\geq 1 \mathrm{a} \leq 5$ salários mínimos & 23 & 60,5 \\
\hline Não souberam responder & 4 & 10,5 \\
\hline \multicolumn{3}{|l|}{ Número de gestações } \\
\hline Primigesta & 18 & 47,4 \\
\hline Multigesta & 20 & 52,6 \\
\hline \multicolumn{3}{|l|}{ Experiência prévia AM } \\
\hline Sim & 12 & 31,5 \\
\hline Não & 26 & 68,4 \\
\hline \multicolumn{3}{|c|}{ Idade gestacional ao nascimento } \\
\hline$\leq 30$ & 29 & 76,3 \\
\hline $31-34$ & 9 & 23,7 \\
\hline \multicolumn{3}{|l|}{ Peso ao nascimento } \\
\hline$<1000 \mathrm{~g}$ & 18 & 47,4 \\
\hline $1000-1500$ & 15 & 39,5 \\
\hline $1501-2500$ & 5 & 13,2 \\
\hline
\end{tabular}

Fonte: Autoras

A Tabela 2 demonstra a frequência das variáveis estudadas de acordo com as semanas de internação dos bebês, sendo analisadas frequências de registro no diário até a sétima semana após o nascimento do bebê. $\mathrm{O}$ uso do "Diário do Bebê" em todas as semanas de internação foi $\geq 3$ dias na semana.

Quanto à média de ordenha diária na primeira semana o predomínio foi de ordenhas menos frequentes $<3$, porém nas demais semanas ocorreu a inversão destes.

Relacionado ao volume ordenhado por dia foi de $\leq 100 \mathrm{ml}$ durante as sete semanas. A frequência de visita da mãe ao bebê na UN foi $\geq 3$ durante todas as semanas de internação. A duração do tempo de ordenha predominante foi entre 16 a 30 minutos. 
Tabela 2 - Frequência das variáveis de acordo com as semanas de internação dos bebês, Londrina, 2011.

\begin{tabular}{|c|c|c|c|c|c|c|c|c|c|c|c|c|c|c|}
\hline \multirow{3}{*}{ Variável } & \multicolumn{14}{|c|}{ Semana de internação } \\
\hline & \multicolumn{2}{|c|}{$1^{\text {a }}$ semana } & \multicolumn{2}{|c|}{$2^{\mathrm{a}}$ semana } & \multicolumn{2}{|c|}{$3^{\text {a }}$ semana } & \multicolumn{2}{|c|}{$4^{\mathrm{a}}$ semana } & \multicolumn{2}{|c|}{$5^{\text {a }}$ semana } & \multicolumn{2}{|c|}{$6^{\text {a }}$ semana } & \multicolumn{2}{|c|}{$7^{\mathrm{a}}$ semana } \\
\hline & $\mathrm{N}$ & $\%$ & $\mathrm{~N}$ & $\%$ & $\mathrm{~N}$ & $\%$ & $\mathrm{~N}$ & $\%$ & $\mathrm{~N}$ & $\%$ & $\mathrm{~N}$ & $\%$ & $\mathrm{~N}$ & $\%$ \\
\hline \multicolumn{15}{|c|}{$\begin{array}{l}\text { Frequência de uso } \\
\text { do diário na semana* }\end{array}$} \\
\hline$<3$ vezes & 06 & 15,8 & 03 & 7,9 & 04 & 10,5 & 03 & 7,9 & 03 & 60,5 & 00 & 00 & 02 & 5,3 \\
\hline$\geq 3$ vezes & 23 & 60,5 & 27 & 71,1 & 23 & 60,5 & 17 & 44,7 & 12 & 7,9 & 11 & 28,9 & 07 & 18,4 \\
\hline \multicolumn{15}{|c|}{$\begin{array}{l}\text { Média de ordenha } \\
\text { realizada por dia* }\end{array}$} \\
\hline$<3$ vezes & 16 & 42,1 & 11 & 28,9 & 06 & 15,8 & 07 & 18,4 & 03 & 7,9 & 03 & 7,9 & 04 & 10,5 \\
\hline$\geq 3$ vezes & 13 & 34,2 & 19 & 50,0 & 21 & 55,3 & 13 & 34,2 & 12 & 31,6 & 08 & 21,1 & 05 & 13,2 \\
\hline \multicolumn{15}{|c|}{$\begin{array}{l}\text { Média de volume } \\
\text { ordenhado por dia* }\end{array}$} \\
\hline$\leq 100 \mathrm{ml}$ & 21 & 72,4 & 17 & 56,7 & 20 & 71,4 & 15 & 75 & 09 & 60 & 09 & 81,9 & 07 & 77,8 \\
\hline$>101 \mathrm{ml}$ & 08 & 21,1 & 13 & 43,4 & 08 & 28,5 & 05 & 25 & 06 & 40 & 02 & 18,2 & 02 & 22,2 \\
\hline \multicolumn{15}{|c|}{$\begin{array}{l}\text { Frequência de visita da } \\
\text { mãe ao bebê na semana* }\end{array}$} \\
\hline$<3$ vezes & 07 & 18,4 & 04 & 10,5 & 00 & 00 & 00 & 00 & 00 & 00 & 00 & 00 & 00 & 00 \\
\hline$\geq 3$ vezes & 30 & 78,9 & 27 & 71,1 & 28 & 100 & 21 & 100 & 15 & 100 & 11 & 100 & 09 & 100 \\
\hline \multicolumn{15}{|c|}{$\begin{array}{l}\text { Média de tempo para } \\
\text { ordenha por dia* }\end{array}$} \\
\hline$\leq 15$ minutos & 04 & 13,8 & 09 & 30 & 09 & 33,3 & 07 & 35 & 05 & 35,7 & 02 & 20 & 02 & 22,2 \\
\hline 16-30 minutos & 12 & 41,4 & 14 & 46,7 & 13 & 48,1 & 12 & 60 & 06 & 42,9 & 06 & 60 & 02 & 22,2 \\
\hline$>30$ minutos & 13 & 44,8 & 07 & 23,3 & 05 & 18,5 & 01 & 05 & 03 & 21,4 & 02 & 20 & 05 & 55,6 \\
\hline
\end{tabular}

Fonte: Autoras

*Obs.: os dados gerados correspondem aos registros realizados pelas mães no diário do bebê (porcentagem válida). A variação do n por semana deve-se à ausência do registro, e/ou à saída da mãe da pesquisa por alta ou óbito do bebê.

Em relação ao estado emocional materno podemos observar que as mães com idade menor ou igual a 19 anos apresentaram predomínio de sentimentos bons durante toda internação do bebê. Outro resultado de destaque relacionado à idade materna consiste em mães com idade igual ou maior 35 anos apresentam na primeira semana de internação predomínio do estado emocional ruim, porém com mudanças para estado emocional bom nas próximas outras semanas de internação do bebê.

Quanto à paridade, na primeira semana as multigestas apresentaram índice elevado de sentimentos ruins $(34,5 \%)$. Em contrapartida as primigestas apresentaram sentimentos bons predominantes durante todo período de internação do bebê.

Com relação à amamentação, as mães que não apresentavam experiência prévia relacionada ao aleitamento materno mantiveram sentimentos bons durante todas as semanas de internação. Já as mães que haviam amamentado pelo menos um filho apresentaram predominância de sentimentos ruins na primeira semana de vida do bebê. Destacase o fato que as mães cujos filhos nasceram com peso menor a $1000 \mathrm{~g}$ apresentaram sentimentos bons durante as sete semanas analisadas. 
Tabela 3 - Estado emocional materno e características obstétricas durante as semanas de internação do filho pré-termo, Londrina, 2011.

\begin{tabular}{|c|c|c|c|c|c|c|c|c|c|c|c|c|c|c|c|c|c|c|c|c|c|c|c|c|c|c|c|c|}
\hline \multirow{4}{*}{$\begin{array}{l}\text { (1) } \\
\\
\text { Estado } \\
\text { emocional }\end{array}$} & \multicolumn{28}{|c|}{ Semana de internação } \\
\hline & \multicolumn{4}{|c|}{$1^{\text {a }}$ semana } & \multicolumn{4}{|c|}{$2^{a}$ semana } & \multicolumn{4}{|c|}{$3^{a}$ semana } & \multicolumn{4}{|c|}{$4^{a}$ semana } & \multicolumn{4}{|c|}{$5^{\text {a }}$ semana } & \multicolumn{4}{|c|}{$6^{a}$ semana } & \multicolumn{4}{|c|}{$7^{a}$ semana } \\
\hline & \multicolumn{2}{|c|}{ Bom } & \multicolumn{2}{|c|}{ Ruim } & \multicolumn{2}{|c|}{ Bom } & \multicolumn{2}{|c|}{ Ruim } & \multicolumn{2}{|c|}{ Bom } & \multicolumn{2}{|c|}{ Ruim } & \multicolumn{2}{|c|}{ Bom } & \multicolumn{2}{|c|}{ Ruim } & \multicolumn{2}{|c|}{ Bom } & \multicolumn{2}{|c|}{ Ruim } & \multicolumn{2}{|c|}{ Bom } & \multicolumn{2}{|c|}{ Ruim } & \multicolumn{2}{|c|}{ Bom } & \multicolumn{2}{|c|}{ Ruim } \\
\hline & $\mathrm{N}$ & $\%$ & $\mathrm{~N}$ & $\%$ & $\mathrm{~N}$ & $\%$ & $\mathrm{~N}$ & $\%$ & $\mathrm{~N}$ & $\%$ & $\mathrm{~N}$ & $\%$ & $\mathrm{~N}$ & $\%$ & $\mathrm{~N}$ & $\%$ & $\mathrm{~N}$ & $\%$ & $\mathrm{~N}$ & $\%$ & $\mathrm{~N}$ & $\%$ & $\mathrm{~N}$ & $\%$ & $\mathrm{~N}$ & $\%$ & $\mathrm{~N}$ & $\%$ \\
\hline \multicolumn{29}{|l|}{$\begin{array}{l}\text { Idade } \\
\text { materna* }\end{array}$} \\
\hline$\leq 19$ anos & 3 & 11,1 & 3 & 11,1 & 7 & 22,5 & 1 & 3,2 & 6 & 21,4 & 1 & 3,7 & 6 & 35,3 & 0 & 0 & 4 & 26,7 & 2 & 13,4 & 3 & 33,4 & 2 & 22,2 & 2 & 28,6 & 1 & 14,3 \\
\hline $20-34$ & 9 & 33,3 & 7 & 25,9 & 10 & 32,3 & 10 & 32,3 & 13 & 46,4 & 5 & 17,8 & 6 & 35,3 & 3 & 17,6 & 7 & 46,7 & 1 & 6,6 & 1 & 11,1 & 2 & 22,2 & 3 & 42,8 & 1 & 14,3 \\
\hline$\geq 35$ & 1 & 3,8 & 4 & 14,8 & 3 & 9,7 & 0 & 0 & 3 & 10,7 & 0 & 0 & 2 & 11,8 & 0 & 0 & 1 & 6,6 & 0 & 0 & 1 & 11,1 & 0 & 0 & 0 & 0 & 0 & 0 \\
\hline \multicolumn{29}{|l|}{ Paridade* } \\
\hline Primigesta & 7 & 24,3 & 6 & 20,6 & 9 & 29,0 & 8 & 25,8 & 13 & 46,4 & 2 & 7,2 & 10 & 58,8 & 2 & 11,7 & 5 & 41,7 & 2 & 16,7 & 3 & 37,5 & 2 & 25,0 & 2 & 22,2 & 2 & 22,2 \\
\hline Multigesta & 6 & 20,6 & 10 & 34,5 & 11 & 35,5 & 3 & 9,7 & 8 & 28,6 & 5 & 17,8 & 5 & 29,5 & 0 & 0 & 4 & 33,3 & 1 & 8,3 & 2 & 25,0 & 1 & 12,5 & 3 & 33,4 & 2 & 22,2 \\
\hline \multicolumn{29}{|c|}{$\begin{array}{l}\text { Experiência } \\
\text { prévia com } \\
\text { AM* }\end{array}$} \\
\hline $\operatorname{Sim}$ & 2 & 7,1 & 6 & 21,4 & 5 & 16,7 & 2 & 6,7 & 4 & 14,3 & 4 & 14,3 & 4 & 23,5 & 0 & 0 & 2 & 14,3 & 1 & 7,1 & 1 & 11,1 & 1 & 11,1 & 1 & 14,3 & 0 & 0 \\
\hline Não & 11 & 39,3 & 9 & 32,2 & 16 & 53,3 & 7 & 23,3 & 17 & 60,7 & 3 & 10,7 & 10 & 58,8 & 3 & 17,7 & 9 & 64,3 & 2 & 14,3 & 5 & 55,6 & 2 & 22,2 & 4 & 57,1 & 2 & 28,6 \\
\hline $\begin{array}{l}\text { Peso de } \\
\text { nascimento }\end{array}$ & & & & & & & & & & & & & & & & & & & & & & & & & & & & \\
\hline$<1000 \mathrm{~g}$ & 7 & 25,0 & 5 & 17,9 & 10 & 32,3 & 4 & 12,8 & 10 & 35,7 & 4 & 14,3 & 8 & 42,1 & 0 & 0 & 6 & 37,5 & 2 & 12,5 & 5 & 55,5 & 0 & 0 & 3 & 37,5 & 1 & 12,5 \\
\hline $1000-1500$ & 4 & 14,3 & 8 & 28,6 & 10 & 32,3 & 5 & 16,2 & 10 & 35,7 & 3 & 10,7 & 5 & 26,3 & 5 & 26,3 & 4 & 25,0 & 3 & 18,8 & 1 & 11,1 & 3 & 33,4 & 2 & 25,0 & 2 & 25,0 \\
\hline $1501-2500$ & 2 & 7,1 & 2 & 7,1 & 2 & 6,4 & 0 & 0 & 1 & 3,6 & 0 & 0 & 1 & 5,3 & 0 & 0 & 1 & 6,2 & 0 & 0 & 0 & 0 & 0 & 0 & 0 & 0 & 0 & 0 \\
\hline
\end{tabular}

Fonte: Autoras

*Excluídos os registros com informações ignoradas. 


\section{Discussão}

Avaliar os preditores que interferem na lactação de mães de bebês nascidos pré-termo se faz necessário. Estudos internacionais mostram que diferentes preditores maternos estão associados à eficácia do aleitamento materno. ${ }^{(16-17)}$ Uma revisão sistemática realizada aponta que comportamentos de parceiros responsivos melhoraram os resultados da amamentação nos contextos da cultura ocidental. ${ }^{(18)}$ Outro estudo transversal realizado no Brasil com 100 puérperas também mostrou associação estatisticamente significativa entre mulheres com companheiro e autoeficácia para o aleitamento materno. ${ }^{(19)}$

A escolaridade, ensino médio completo ou mais também está associada à prática do aleitamento materno exclusivo conforme resultados de uma pesquisa realizada nos Estados Unidos com 190 mulheres. ${ }^{(16)}$ Como resultados de uma revisão sistemática com metanálise realizado nos EUA, os modelos de efeitos aleatórios para o início da amamentação foi mais alto para educação materna alta $v s$. baixa (RR 2,28 [IC 95\% 1,92$2,70]$ ) e relação díade vs. não (RR 2,01 [IC 95\% 1,38-2,92]) com resultados semelhantes também para a continuação da amamentação. ${ }^{(16)}$

Referente à idade gestacional do recémnascido destaca-se o nascimento anterior a 30 semanas e peso inferior a $1000 \mathrm{~g}$ nesta pesquisa. Instrumentos são essenciais para a promoção e facilitação do processo de aleitamento materno do recém-nascido pré-termo durante a hospitalização. Um estudo realizado em Utah mostrou associação estatisticamente significativa em mães que relataram iniciar e alimentar apenas com o leite materno no hospital e receber um número de telefone para pedir ajuda com a amamentação tiveram uma menor prevalência de interrupção do aleitamento materno antes de dois meses. ${ }^{(20)}$

A frequência do uso do diário foi maior ou igual a três vezes/semana, durante as sete semanas de coleta de dados. Uma pesquisa sobre o significado do diário para a mãe demonstrou que o uso do mesmo favoreceu o enfrentamento das famílias em relação à prematuridade do filho, serviu de subsídio para estarem mais informadas sobre as condições do bebê e contribuíram com o estímulo do AM. ${ }^{(15)}$

A média de ordenha realizada por dia na primeira semana de internação foi menor que três, sendo nas semanas seguintes, maior ou igual a três. A ordenha precoce iniciada no período em que a mãe ainda se encontra hospitalizada é de suma importância para a manutenção do AM. Sendo necessária a preocupação dos profissionais para que a técnica da ordenha inicie-se precocemente independe do estado do recém-nascido, garantido deste modo o momento da apojadura. ${ }^{(20)}$

A produção láctea está diretamente relacionada à frequência de ordenha. A ordenha sistematizada é necessária para a manutenção da lactação, embora possa ser em alguns momentos considerada cansativa e desgastante pelas mães. ${ }^{(21)}$ No serviço pesquisado as mães são orientadas a ordenhar pelo menos a cada três horas visando o esvaziamento completo das mamas, conforme recomenda a literatura. ${ }^{(21)}$ No entanto, percebe-se neste estudo que a ordenha passa a ser realizada de modo sistematizado a partir da segunda semana de internação do filho pré-termo. Talvez fatores como a preocupação com a condição de saúde da criança e o início da vivência do processo de internação possam ser os responsáveis por este fato. Em contrapartida, no decorrer da internação, os pais passam a se familiarizar mais com o ambiente e os profissionais, o que colabora com o aumento da frequência de ordenha.

No que tange à média de volume ordenhado registrado no diário foi menor ou igual a $100 \mathrm{ml}$ ao dia durante as sete semanas. O ambiente e a rotina hospitalar podem trazer desconforto, sendo que a infraestrutura e as regras rígidas, horário e local de amamentação podem gerar ansiedade a essas mulheres, prejudicando e até mesmo postergando o inicio do aleitamento. ${ }^{(22)}$ Permitir e estimular a ordenha mecânica na beira do leito e a proximidade com seu bebê pode aumentar a estimulação de hormônios responsáveis na produção de leite. ${ }^{(21-23)}$ 
O tempo predominante de ordenha foi de 16 a 30 minutos. A duração da ordenha nos primeiros dias pós-parto deve ser de 10 a 15 minutos, sendo que após a apojadura deve ocorrer até o esvaziamento completo da mama, o que pode levar cerca de 20 a 30 minutos. ${ }^{(24)}$

Durante toda a internação, a visita da mãe ao bebê foi maior ou igual a três/semana. Mesmo após o parto, com o estado de saúde fragilizado, as mães sempre estiveram presentes. Esta realidade pode ser devido ao acolhimento da equipe multiprofissional e ao trabalho de acompanhamento do projeto "Uma rede de apoio à família prematura".(14) Por ser um hospital referência para todo o Norte do Paraná, existem muitas mães de outras cidades. É importante destacar que o hospital conta com um atuante serviço de assistência social, responsável por auxiliar estas famílias, disponibilizando passes ou agendando transporte com ambulância ou carros da saúde. Estas mães são encaminhadas, se desejarem, para uma casa de apoio, próxima ao hospital. Também é fornecido refeição às mães durante todo o período em que permanecerem nas unidades.

A preocupação em auxiliar a mãe a permanecer no setor é observada em toda a equipe, que ajuda a família a identificar redes de apoio, inclusive para deixar os outros filhos enquanto visitam o bebê. Destaca-se a importância da equipe multiprofissional em mobilizar e fortalecer os recursos das famílias e identificar grupos de apoio.

Outro fator que atua positivamente na permanência das mães no setor é a utilização da primeira etapa do Método Canguru (MC). Todas as mães são encorajadas e auxiliadas a realizá-lo tão logo o bebê esteja clinicamente estável. Uma revisão sistemática sobre a influência do MC na morbimortalidade de bebês pré-termos identificou importante impacto do Método sobre as taxas de aleitamento materno exclusivo na alta e nos primeiros seis meses de vida de recém-nascidos baixo peso. ${ }^{(25)} \mathrm{O}$ efeito desse modelo de assistência humanizada, que preconiza a presença da mãe junto ao bebê, valorizando e empoderando a mulher para o cuidado e realização do contato pele a pele são fundamentais para desfechos como este. ${ }^{(26)}$
Em relação ao estado emocional materno, as mães com idade menor ou igual a 19 anos apresentaram predomínio de sentimentos bons durante toda internação do bebê, enquanto as mães com idade igual ou maior 35 anos apresentam na primeira semana de internação predomínio do estado emocional ruim.

O medo da perda iminente e do desconhecido pode transformar o momento do nascimento de um filho, em uma vivência de angústias, dúvidas e incertezas sobre o futuro próximo e distante do bebê. A vivência do processo de hospitalização constitui uma experiência estressante para os pais e a separação do filho pode ser muito difícil, fazendo aflorar diversos sentimentos e emoções como tristeza, medo, pena, culpa, raiva, impotência, decepção, sensação de perda, frustração, vazio, esperança e outros. ${ }^{(26-28)}$

Enquanto as multigestas e mulheres com experiências prévias relacionadas à amamentação apresentaram índice elevado de sentimentos ruins, já as primigestas e as que nunca haviam amamentado apresentaram sentimentos bons predominantes durante todo período de internação do bebê, bem como as mães cujos filhos nasceram com peso menor a $1000 \mathrm{~g}$ nesta pesquisa.

Mulheres multíparas podem ser consideradas experientes no processo de amamentação e não receberem adequado preparo pelos profissionais da saúde. Uma pesquisa realizada no Japão relata que as mães gastaram $20 \%$ do período de internação pós-parto para atividades relacionadas ao AM. É necessário apoio individual, especialmente para as primíparas, que passaram mais tempo do que as multíparas para atividades relacionadas ao AM no quarto dia pós-parto. ${ }^{(29)}$

Evidência robusta para o efeito da experiência anterior de amamentação na amamentação subsequente estápresenteemumarevisãosistemática realizada na China, pois a curta duração anterior da amamentação e a experiência insatisfatória afetaram negativamente a amamentação subsequente. Consultores em aleitamento materno precisam fornecer intervenções personalizadas para as mães, de acordo com a experiência anterior detalhada 
de amamentação, a fim de melhorar o início da amamentação e a duração da amamentação. ${ }^{(30)}$

A amamentação deve ser assistida de forma individualizada e sistematizada pelo enfermeiro que permita a reflexão junto a todas as nutrizes a respeito do vivenciar a amamentação, das dúvidas, facilidades e dificuldades.

A amamentação ocorre de maneira única em cada filho da mulher sendo imprópria a rotulação de experiente ou inexperiente em amamentar para a assistência à puérpera. Sendo assim, é necessária uma atuação profissional individualizada, independente do número de filhos, que permita a reflexão junto à nutriz a respeito do vivenciar a amamentação. ${ }^{(8)}$ Uma revisão sistemática realizada em 2019 mostrou que a intervenção baseada no apoio é provavelmente uma maneira eficaz de promover a amamentação. ${ }^{(31)}$

Com o aumento da prematuridade e avanço das tecnologias para garantir a sobrevida dos bebês pré-termos, avaliar os fatores que interferem na lactação destas mães se faz necessário, uma vez que há na literatura lacunas sobre o processo de amamentar estes bebês.

Os dados analisados neste estudo buscam um olhar diferenciado para a amamentação do bebê pré-termo. A proposta de acompanhar a produção láctea, realização da ordenha e o estado emocional materno a partir de registros da própria mãe em um material oferecido para esse fim, o "Diário do Bebê", conferem a inovação da pesquisa, apresentando uma nova estratégia utilizada visando o sucesso dessa amamentação.

\section{Conclusão}

Considera-se que os objetivos do estudo foram alcançados ao descrever as variáveis envolvidas na manutenção da lactação da mãe do bebê internado em uma UN registradas em um diário utilizado pela mãe.

Pode-se identificar que na primeira semana a frequência de ordenha foi baixa, bem como a média de volume ordenhado, porém próximo da alta esta frequência de ordenha elevou-se. Sabendo-se que a ordenha é de suma importância para a manutenção da produção láctea nos casos de impossibilidade de sucção do bebê, os dados descritos nos diários possibilitam que os profissionais que atuam junto ao binômio possam elaborar estratégias que garantam a ordenha precoce e frequente.

O estado emocional materno apresentou labilidade de acordo com a característica analisada. Sabendo que o estado emocional pode interferir na produção láctea, faz-se necessário novos estudos que possam aprofundar estas discussões.

No entanto, este estudo apresenta limitações ao número pequeno de participantes, dificultando a realização de testes estatísticos. Também, recomenda-se uma pesquisa com abordagem qualitativa com as mães que não utilizaram o diário, apesar de inicialmente terem aceitado participar da pesquisa, para compreensão mais aprofundada dos motivos que as levam a optar pelo não registro no diário e a falta de estímulo para realização das ordenhas para manutenção da lactação.

Como ponto forte desse estudo, o acompanhamento da produção láctea, realização da ordenha e o estado emocional materno a partir de registros da própria mãe em um instrumento oferecido para esse fim, o "Diário do Bebê", conferem a inovação ao estudo, apresentando uma nova estratégia utilizada visando o sucesso dessa amamentação na prática da Unidade Neonatal. Portanto, o incentivo para o registro no Diário pode ser um recurso auxiliar para a manutenção da produção láctea.

\section{Referências}

1 World Health Organization. Guideline: protecting, promoting and supporting breastfeeding in facilities providing maternity and newborn services. Geneva: World Health Organization; 2017.

2 Victora CG, Bahl R, Barros AJD, França VA, Horton S, Krasevec J, et al. Brastfeeding in the 21st century: epidemiology, mechanisms, and lifelong effect. The Lancet. 2016 Jan; 387(10017):475-90. doi: 10.1016/s01406736(15)01024-7. 
3 Mahesh PKB, Gunathunga MW, Arnold SM, Jayasinghe C, Pathiana S, Makarim MF, et al. Effectiveness of targeting fathers for breastfeeding promotion: systematic review and meta-analysis. BMC Public Health. 2018 Sept; 18(1140). doi: 10.1186/s12889-018-6037-x.

4 Bellù $\mathrm{R}$, Condò $\mathrm{M}$. Breastfeeding promotion: evidence and problems. Pediatr Med Chir. 2017 June 28; 39(2).

5 Wojcicki JM, Heyman MB, Elwan D, Lin J, Blackburn E, Epel E. Early exclusive breastfeeding is associated with longer telomeres in Latino preschool children. Am. J. Clin. Nutr. 2016 Aug; 104(2):397-405. doi: 10.3945/ajcn.115.115428.

6 Renfrew MJ, McCormick FM, Wade A, Quinn B, Dowswell T. Support for healthy breastfeeding mothers with healthy term babies. Cochrane Database Syst Rev. 2012 May 16;5(5):CD001141.

7 Brusco TR, Delgado SE. Caracterização do desenvolvimento da alimentação de crianças nascidas pré-termo entre três e 12 meses. Rev. CEFAC [Internet]. 2014 maio [citado 2015 feb 10]; 16(3):917-28. Disponível em: http://www.scielo.br/scielo.php?script=sci arttext\&pid=S1516-18462014000300917\&ln $\mathrm{g}=\mathrm{pt} \& \mathrm{t} \operatorname{lng}=\mathrm{pt}$

8 Gianni ML, Bezze EN, Sannino P, Baro M, Roggero P, Muscolo S, et al. Maternal views on facilitators of and barriers to breastfeeding preterm infants. BMC Pediatr [Internet]. 2018 Aug [cited 2019 jul. 5]; 18(1). Available from: https://bmcpediatr.biomedcentral.com/ articles/10.1186/s12887-018-1260-2\#Decs

9 Boucher CA, Brazal PM, Graham-Certosini C, Carnaghan-Sherrard K, Feeley N. Mothers' breastfeeding experiences in the NICU. Neonatal Netw [Internet]. 2011 [cited 2019 jul. 5]; 30(1):21-8. Available from: https://connect. springerpub.com/content/sgrnn/30/1/21

10 Brumbaugh JE, Colaizy TT, Saha S, Van Meurs KP, Das A, Walsh MC, et al. Oral feeding practices and discharge timing for moderately preterm infants. Early Hum. Dev. 2018 May; 120:46-52. doi: 10.1016/j. earlhumdev.2018.04.001.
11 Ikonen R, Paavilainen E, Kaunonen M, Ikuta L, Zukowsky K. Preterm Infants' Mothers' Experiences With Milk Expression and Breastfeeding. Adv Neonatal Care. 2015 Dec; 15(6):394-406. doi: 10.1097/ ANC.0000000000000232.

12 Raines DA. Mothers' stressor as the day of discharge from the NICU approaches. Adv Neonatal Care. 2013;13(3):181-7. doi: 10.1097/ANC.0b013e318285fa2a.

13 Nyqvist KH, Häggkvist AP, Hansen MN, Kylberg E, Frandsen AL, Maastrup R, et al. Expansion of the baby-friendly hospital initiative ten steps to successful breastfeeding into neonatal intensive care: expert group recommendations. JHum Lact. 2013;13(3):3009. doi: 10.1177/0890334413489775.

14 Bengozi TM, Souza NDH, Rossetto EG, Radigonda B, Hayakawa LM, Ramalho DP. Uma rede de apoio à família do prematuro. Ciênc Cuid Saúde. 2010;9(1):155-60. doi: 10.4025/cienccuidsaude.v9i1.10565.

15 Leite CCdP, Souza SNDH, Rossetto E, Pegoraro LGdO, Jacinto VCB. O diário do bebê para a mãe de prematuro: apoiando o cuidado centrado na família. Reva Enfer UERJ. 2016 Jul;24(1):8664. doi: 10.12957/reuerj.2016.8664.

16 Cohen SS, Alexander DD, Krebs NF, Young BE, Cabana MD, Erdmann $\mathrm{P}$, et al. Factors associated with breastfeeding initiation and continuation: a meta-analysis. J. Pediatr. 2018 Dec;203(21):190-6. doi: 10.1016/j. jpeds.2018.08.008.

17 Rethy JA, Gallo S, Doig C, Brady J,Goodfriend, D. Sociodemographic predictors of exclusive breast-feeding among low-income women attending a special supplemental nutrition program for women, infants, and children (WIC) programme. Public Health Nutr. 2019 Feb; 22(9):1667-74. doi: 10.1017/ S1368980019000119.

18 Davidson EL, Ollerton RL. Partner behaviours improving breastfeeding outcomes: An integrative review. Women and Birth. 2019 June; 33(1):15-23. doi: 10.1016/j. wombi.2019.05.010. 
19 Abreu LM, Filipini R, Alves BdCA, Veiga GL, Fonseca FLA. Evaluation of breastfeeding self-efficacy of puerperal women in shared rooming units. Heliyon. 2018 Oct; 4(10):900. doi: 10.1016/j.heliyon.2018.e00900.

20 Schliep KC, Denhalter D, Gren H, PanushkaKA, Varner W, Singh P, et al. Factors in the Hospital Experience Associated with Postpartum Breastfeeding Success. Breastfeed Med. 2019 June;14(5). doi: 10.1089/bfm.2018.0039.

21 Zulin NE, Tacla TGM, Souza NDH, Monteiro ATA, Ferrari AP. Vivência de mães de prematuros no processo de translactação. Semina: Ciênc. Biol. Saúde. 2015;36(1Supl). doi: 10.5433/1679-0367.2014v35n2p363.

22 Rodrigues AP, Martins EL, Trojahn TC, Padoin SM de M, de Paula CC, Tronco CS. Manutenção do aleitamento materno de recém-nascidos pré-termo: revisão integrativa da literatura. Rev. Eletr. Enf. 2013;15(1):25364. doi: 10.5216/ree.v15i1.17067.

23 Maciel IVL, Almeida CS, Braga PP. Breastfeeding in the context of prematurity: the maternal speech. J Nurs UFPE. 2014 maio; 8(5):1178-84. doi: 0.5205/reuol.5863-505311-ED.0805201411.

24 Gurgel AKC, Monteiro AI. Difficulties related to breastfeeding in premature newborn: integrative literature review. J Nurs UFPE. 2013;7(esp):7181-7. doi: 10.5205/1981-8963v7i12a12391p7181-7187-2013.

25 Conde-Agudelo A, Díaz-Rosello JL. Kangaroo mother care to reduce morbidity and mortality in low birthweight infants. Cochrane Database Syst Rev. 2016;8:2772. doi: 10.1002/14651858. CD002771.pub4.

26 Testoni TT, Aires LCdP. O método canguru como um veículo para o empoderamento materno. Refacs. 2018;6(2):611-9. doi: 10.18554/refacs.v6i0.2957.

27 Zani AV, Golias ARC, Martins STF, Parada CMGL, Marcon SS, Tonete VLP. Feelings experienced by the family of an at-risk newborn infant: integrative literature review. J Nurs UFPE. 2013; 7(1):269-78. doi: 10.5205/ reuol.3049-24704-1-LE.0701201335.
28 Zani AV, Tonete VL, Parada CMGL. Maternal representations about the provision of care to newborns at risk: a collective discourse. Online Brazilian Journal of Nursing. 2014;13(3):32131. doi: doi.org/10.5935/1676-4285.20144328.

29 Mitsui Y, Saito I. Mothers' BreastfeedingRelated Durations and Nursing Management During the Early Postpartum Period in a Mixed Hospital Ward with an Obstetrics Department: A Prospective Observational Study. Kobe J. Med. Sci [Internet]. 2019 Jan [cited 2019 jun 5]; 64(5):E160-E9. Available from: http:// www.med.kobe-u.ac.jp/journal/contents/64/ E160.pdf

30 Huang Y, Ouyang YQ, Redding SR. Previous breastfeeding experience and its influence on breastfeeding outcomes in subsequent births: a systematic review. Women and Birth. 2019 Aug; 32(4):303-9. doi: 10.1016/j. wombi.2018.09.003.

31 Cheng LY, Kit-han Mo. The effect of homebased intervention with professional support on promoting breastfeeding: a systematic review. Int J One Health. 2019 Jun; 64:9991014. doi: 10.1007/s00038-019-01266-5. 